\title{
A novel chimeric CYP11B2/CYP11B1 combined with a new p.L340P CYP11B1 mutation in a patient with 110HD: case report
}

\author{
Lian Duan ${ }^{1 \dagger}$, Rufei Shen ${ }^{1 \dagger}$, Lingyu Song $^{1}$, Yong Liao ${ }^{2}$ and Hongting Zheng ${ }^{1 *}$ (B)
}

\begin{abstract}
Background: $11 \beta$-Hydroxylase deficiency $(11 \mathrm{OHD})$ is a common form of congenital adrenal hyperplasia that has been shown to result from inactivating CYP11B1 mutations, and pathogenic CYP11B2/CYP11B1 chimeras contribute to a minority of cases. Heterozygote cases (chimeras combined with missense mutation) are very rare, and genetic analysis of these cases is difficult.

Case presentation: We describe an 11OHD patient presenting with precocious pseudopuberty and hypokalemia hypertension who harbored a chimeric CYP11B2/CYP11B1 with a novel breakage point located at g.9559-9742 of CYP11B2. Interestingly, the other allele exhibited a new mutation, p.L340P, in CYP11B1. Bioinformatics and molecular dynamics simulation indicated that P.L340P decreased the stability and changed the surface configuration of $11 \beta$ hydroxylase, indicating a disease-causing mutation. Further pedigree study, PCR and next-generation sequencing indicated that the proband carried both the chimera and p.L340P, and coexistence of the two increased the severity of $110 H D$ in this family. After treatment with combined medications, blood pressure and clinical parameters improved.
\end{abstract}

Conclusions: Our results suggest that chimera screening and CYP11B1 mutation screening should be simultaneously conducted, and pedigree study is necessary.

Keywords: Chimeric CYP11B2/CYP11B1, Missense mutation, 11ß-hydroxylase deficiency

\section{Background}

Congenital adrenal hyperplasia $(\mathrm{CAH})$ is one of the most common inheritable metabolic disorders and is characterized by virilization, precocious pseudopuberty and accelerated skeletal maturation, progressing in some cases to severe dehydration, shock, and even death [1, 2]. An autosomal recessive disorder, $\mathrm{CAH}$ is caused by mutations in genes encoding important enzymes or cofactors in the steroidogenesis pathway [3]. One common variant of $\mathrm{CAH}$ is $11 \beta$-hydroxylase deficiency (11OHD), driven by CYP11B1 inactivating mutations clustered in exons 2, 6, 7 and 8 [4], and approximately 148 mutations have been reported in the Human Gene Mutation

\footnotetext{
* Correspondence: fnf7703@hotmail.com

${ }^{\dagger}$ Equal contributors

'Department of Endocrinology, Xinqiao Hospital, Third Military Medical

University, Chongqing 400037, China

Full list of author information is available at the end of the article
}

Database website. However, CYP11B1 mutations may also occur as a result of aberrant incorporation/chimerism of the gene with the highly homologous aldosterone synthase $(C Y P 11 B 2)$ gene sequence. These $C Y P 11 B 2 / C Y P 11 B 1$ chimeric genes are relatively rare, with breakpoints dispersively distributed, and have been speculated to be pathogenic because of a loss of function in the zona fasciculate/reticularis, despite maintained function in the zona glomerulosa [5-9]. In a few cases, missense mutation and chimera have been found in the same individual [6]. However, it is difficult to evaluate the precise contributing mechanism.

In the present paper, we report an 11OHD case who harbored a chimeric $C Y P 11 B 2 / C Y P 11 B 1$ on one allele with a breakpoint range (g.9559-9742) in the CYP11B2 gene in the junctional zone of exon 6 and intron 6 . Interestingly, the other allele held a novel mutation, $\mathrm{p}$. 
L340P, and bioinformatics and molecular dynamics simulation indicated that this was a disease-causing mutation. We then performed a pedigree analysis, which revealed that both the missense mutation and chimera were synergistically pathogenic in the proband.

\section{Case presentation}

A 14-year-old boy (46, XY) was admitted in September 2015 because of precocious puberty and recurrent episodes of periodic paralysis without special intervention. His sexual development, such as pubic hair, laryngeal prominence and spermatorrhea, occurred earlier than that in peers. He was born after an uneventful full-term pregnancy to a non-consanguineous healthy couple of Chinese origin with no family history of congenital adrenal hyperplasia. He was taller than boys of the same age until he was 12 years old. In addition, axillary hair and laryngeal prominence had appeared at 7 years old, and spermatorrhea and pubic hair growth had occurred at age 9. Physical examination showed hypertension $(143 / 106 \mathrm{mmHg})$, slightly black skin, and external genitalia maturation (pubic hair and axillary hair at stage 5 assessed by Tanner classification and an estimated testicular volume of $25 \mathrm{ml}$ on both sides, as measured by an orchidometer), which confirmed precocious puberty (Fig. 1a). Laboratory data in the absence of medication showed decreased plasma potassium and cortisol but elevated levels of plasma 17 hydroxyprogesterone (17OHP), androstenedione, adrenocorticotrophic hormone $(\mathrm{ACTH})$, and uric 17 ketosteroid (17 KS) (Additional file 1: Table S1). Additionally, serum aldosterone (ALD) was normal with low rennin activity, and renal, liver, metadrenaline, normetadrenaline and thyroid function, were all normal (Additional file 1: Table S1). Radiation imaging showed accelerated bone aging (over 18 years according to the Greulich and Pyle Atlas) (Fig. 1b) and bilateral adrenal hyperplasia (Fig. 1c). The hypokalemic hypertension, elevated 17-OHP and androstenedione were all suggestive of an 11OHD diagnosis. Interestingly, low urine osmotic pressure and specific gravity could not be significantly elevated by water deprivation and a desmopressin test. This phenomenon is consistent with nephrogenic diabetes insipidus that may be due to long-term hypokalemia (Additional file 1: Table S1).

Given this clinical presentation, we then sought to confirm whether it was indeed a case of 11OHD. To find the possible genetic pathogenic mechanism, we conducted sequencing of the nine exons and flanking sequencing of the CYP11B1 gene and identified the novel mutation p.L340P (CYP11B1mut) (Fig. 1d). Bioinformatics and molecular dynamics simulation indicated that CYP11B1mut altered the free energy, and change the stability and conformation of the protein by driving the uncoiling of a partial helical structure into a loop structure, leading to positive group migration and pocket structure enlargement (Additional file 2: Table S2, Figure 1e, f), suggesting that the mutation could be pathogenic. However, his mother did not carry this pathogenic mutation (Fig. 1g), indicating the proband only carried the point mutation on one allele, with the pathogenicity of the other allele unclear. Since 11OHD is understood to be an autosomal recessive condition, we then investigated the genotype of the patient in greater detail. QPCR of the sequence around the mutation of the proband showed that the copy number was half the normal number, which indicated a fragment deletion, and this was also found in his mother (Additional file 3: Figure S1). Targeted next-generation sequencing of the patient genome then revealed a large fragment deletion that included exons 1 to 6 of CYP11B1 (Additional file 3: Figure S1). To validate the deletion, we conducted PCR in proband with mixed oligonucleotide primers that have been previously reported [8] (a forward primer complementary to the $C Y P 11 B 2$ sequence and a reverse primer complementary to the $C Y P 11 B 1$ sequence) and confirmed a chimeric $C Y P 11 B 2 / C Y P 11 B 1$ gene. Interestingly, extended familial analysis revealed that the chimera was also present in his mother, uncle and grandfather (Fig. 1h), and multiple sequence alignment revealed that the breakpoint was located at g.9559-9742 of CYP11B2, considering the cytosine nucleotide base $(C)$ of rs6391 in this family with 3 single nucleotide polymorphisms (SNPs) (Fig. 1i). Our results suggested that the proband harbored a compound heterozygosity for a chimeric $C Y P 11 B 2 / C Y P 11 B 1$ gene combined with a novel missense mutation p.L340P (Fig. $1 \mathrm{j}$ and k, Additional file 4: Figure S2) as two contributors to pathogenicity.

Since the blood pressure of the patient was poorly controlled by hydrocortisone, the proband was treated with a combination of dexamethasone $(0.75 \mathrm{mg} /$ everyday) and levamlodipine (2.5 mg/everyday) for 20 months. After treatment, clinical parameters, such as blood pressure, 17OHP and ACTH decreased, and the volume of the adrenal glands was reduced (Additional file 1: Table S1, Additional file 5: Figure S3). Main methods used in the study are summarizing in Additional file 6. This study was approved by the ethics committees of Xinqiao Hospital, Third Military Medical University, and informed consent was obtained from the patient's mother (Additional file 7).

\section{Discussion and conclusion}

$11 \mathrm{OHD}$ presents with decreased cortisol and corticosterone synthesis due to impaired conversion of 11-deoxycortisol and 11-deoxycorticosterne (DOC) to cortisol and corticosterone, respectively, and excess androgens due to DOC accumulation [10]. Although DOC was not detected, all of the classical 11OHD symptoms, 


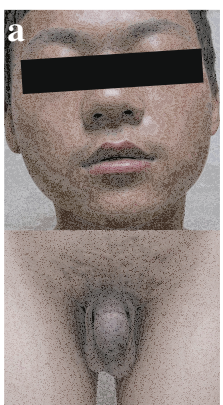

d

270

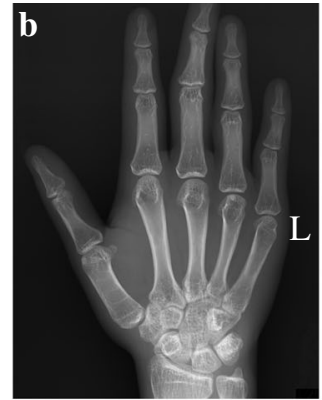

280
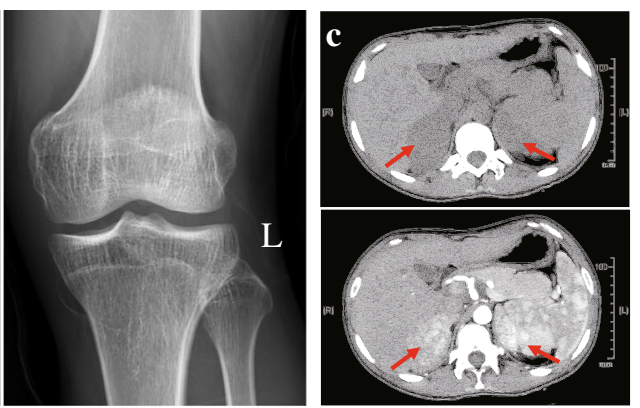

\section{f}

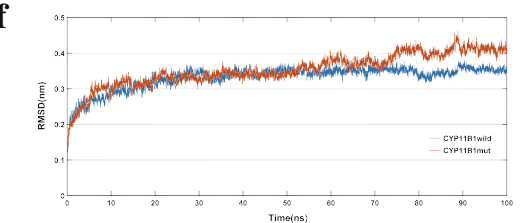

$\mathbf{g}$

$\stackrel{270}{27}$ 

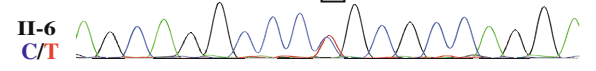

h

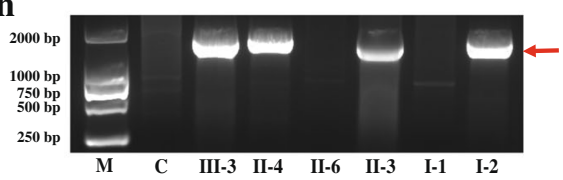

CYP11B1mut

CYP11B1wild

i

$\Delta$

$\Delta$ GCGCCAGGAGAGCCTGGCCGCCGCAGCCAGCATCAGT GAACATCCCCAGAAGGCAACCACCG $9511^{\circ}$ CYP11B2-Fathe CYP11B2-Mother CGACGTGCAGCAGAIICDIGCGCCAGGAGAGCCTGGCCGCCGCAGCCAGCATCAGTGAACATCCCCAGAAGGCAACCACCG 273 CYP11B2/CYP11B1-Proband OEACGTGCAGCAGAICOIGCGCCAGGAGAGCCTGGCCGCCGCAGCCAGCATCAGTGAACATCCCCAGAAGGCAACCACCG 631

CYP11B1-Mother CAACGTGCAGCAGGCCDIIGCGCCAGGAGAGCCTGGCCGCCGCAGCCAGCATCAGTGAACATCCCCAGAAGGCAACCACCG 339

CYP11B1-Father CAACGTGCAGCAGGCCCCGCGCCAGGAGAGCCTGGCCGCCGCAGCCAGCATCAGTGAACATCCCCAGAAGGCAACCACCG 335

CYP11B1-NCBI CAACGTGCAGCAGGCCD而GCGCCAGGAGAGCCTGGCCGCCGCAGCCAGCATCAGTGAACATCCCCAGAAGGCAACCACCG 9069

CYP11B2-NCBI AGCTGCCCTTGCTGCGEGCGGCCCTCAAGGAGACCTTEGGTGGGTGCTGGCTGAGGCCTCCCTGTGGCCCTGGCCCCCT 9599 CYP11B2-Father AGCTGCCCTTGCTGCGEGCGGCCCTCAAGGAGACCTTGAGGTGGGTGCTGGCTGAGGCCTCCCTGTGGCCCTGGCCCCCT 351 CYP11B2-Mother AGCTGCCCITGCTGCGEGCGGCCCTCAAGGA GACCTTGAGGTGGGTGCTGGCTGAGGCCTCCCTGTGGCCCTGGCCCCCT 353 CYP11B2/CYP11B1-Proband AGCTGCCCTIGCTGCGEGCGGCCCTCAAGGAGACCTTGAGGIGGGTGCTGGCTGAGGCCTCCCTGTGGCCCTGGCCCCCT 711 CYP11B1-Mother AGCTGCCCTTGCTGCGTGCGGCCCTCAAGGAGACCTTG GGGTGGGTGCTGGCTGAGGCCTCCCTGTGGCCCTGGCCCCCT 419 CYP11B1-Father AGCTGCCCTTGCTGCGTGCGGCCCTCAAGGAGACCTTECGGTGGGTGCTGGCT GAGGCCTCCCTGTGGCCCTGGCCCCCT 415 CYP11B1-NCBI AGCTGCCCTTGCTGCGIGCGGCCCTCAAGGAGACCTTGDGGIGGGTGCTGGCTGAGGCCTCCCTGTGGCCCTGGCCCCCT 9149 Exon $6 \longleftrightarrow$ Intron 6

CYP11B2-NCBI GCTGGAGAGCAGCCCCCACTGGGTGGTGGCAGACAGAATCTGGGGCTGATAAACAGCGTCACCCAGCAGCCCATTCCCCT 9679 CYP11B2-Father GCTGGAGAGCAGCCCCCACIGGGTGGTGGCAGACAGAATCTGGGGCTGATAAACAGCGTCACCCAGCAGCCCATTCCCCT 431 CYP11B2-Mother GCTGGAGAGCAGCCCCCACTGGGTGGTGGCAGACAGAATCTGGGGCTGATAAACAGCGICACCCAGCAGCCCATTCCCCT 43.3 CYP11B2/CYP11B1-Proband GCTGGAGAGCAGCCCCCACTGGGTGGTGGCAGACAGAATCTGGGGCT GATAAACAGCGTCACCCAGCAGCCCATTCCCCT 79

CYP11B1-Mother GCTGGAGAGCAGCCCCCACTGGGTGGTGGCAGACAGAATCTGGGGCTGATAAACAGCGTCACCCAGCAGCCCATTCCCCT 499 CYP11B1-Father GCTGGAGAGCAGCCCCCACTGGGIGGTGGCAGACAGAAICTGGGGCT GATAAACAGCGICACCCAGCAGCCCATTCCCCT 495 CYP11B1-NCBI GCTGGAGAGCAGCCCCCACTGGGTGGTGGCAGACAGAATCTGGGGCTGATAAACAGCGTCACCCAGCAGCCCATTCCCCT 9229

CYP11B2-NCBI GCACCTGCTCTTCCTCCCCCTCAAGGACAGGGAGCTCTTCT ICCTCTGGAATCCCTCTTCAACACCCTGGGGATTAACGT CYP11B2-Father GCACCTGCTCTTCCTCCCCCTCAAGGACAGGGAGCTCTTCTIOCTCTGGAATCCCTCTTCAACACCCTGGGGATTAACGT 51 CYP11B2-Mother GCACCIGCICTICCTCCCCCTCAAGGACAGGGAGCTCIICTMCCTCTGGAATCCCTCTTCAACECCCTGGGGATTAACGT 51 CYP1B2/CYP1B1-roband GCACCIGCICITCCICCCCCICAAGGACAGGGAGCICIICI IOCTCIGGAAICCCICIICAACECCCIGGGGATTAACGT 87 CYP11B-Mother GCACCTGCTCTTCCTCCCCCTCAAGGACAGGGAGCTCTICTHCCTCTGGAATCCCTCTTCAAGECCCTGGGGATTAACGT 579 CYP11B1-Father GCACCTGCTCTTCCTCCCCCTCAAGGACAGGGAGCTCTTCTMCCTCTGGAATCCCTCTTCAACECCCTGGGGATTAACGT 575 CYP1B1-NCBI GCACCIGCTCTTCCTCCCCCTCAAGGACAGGGAGCICTICITTCICTGGAATCCCTCITCAACECCCTGGGGATTAACGI 9309

j

CYP11B2 CYP11B1 L340P

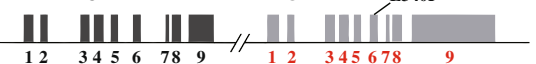

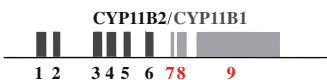

k

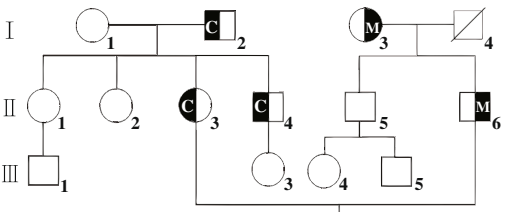

Proband $\rightarrow \mathrm{CM}_{2}$

Fig. 1 (See legend on next page.) 
(See figure on previous page.)

Fig. 1 Clinical data and gene mutations of the proband and his family. a Physical examination results for the proband. b Radiograph of left wrist and knee. c Abdominal CT scan of the proband, with and without contrast enhancement. Arrow shows the bilateral adrenal lesions. $\mathbf{d}$ The sequencing chromatogram near the mutation in proband. Box indicates the mutation location. e Three-dimensional structure of wild-type CYP11B1 (CYP11B1wild) and the CYP11B1 mutant (CYP11B1mut) after 100 ns simulation based on bioinformatics and the molecular dynamics simulation. $\mathbf{f}$ Chart of root mean square deviation (RMSD) calculations for both CYP11B1wild and CYP11B1mut in the molecular dynamics simulation. $\mathbf{g}$ The sequencing chromatogram on both sides of the mutation in the proband's parents. The box indicates the mutation location. $\mathbf{h}$ Electrophoretogram (on a 1\% agarose gel) of PCR products obtained from control, proband and proband relatives using mixed primers. The arrow shows the 1649 base pair DNA fragment of the CYP11B2/CYP11B1 chimera. i The nucleotide sequence neighboring the crossover site of the chimeric CYP11B2/CYP11B1 gene based on multiple sequence alignment. The asterisk, triangle and red line represent a single nucleotide polymorphism, P.L340P and the possible breakpoint regions, respectively. $\mathbf{j}$ A schematic representation of genotype for the proband, the CYP11B2 gene (exons displayed as black boxes), the CYP11B1 gene (exons displayed as gray boxes) and the hybrid gene with the breakpoint localized in the junctional zone of exon 6 and intron 6 . $\mathbf{k}$ The pedigree of all family members investigated for the missense mutation $(M)$ and the chimeric gene $(C)$

precocious pseudopuberty, accelerated skeletal maturation and hypokalemia hypertension [11, 12], were present in our patient. After treatment with the combined medication regimen described above, the clinical symptoms of the patient improved and the adrenal gland volume was reduced.

From our investigation of the genetic contribution to pathogenesis, this patient was found to harbor a chimeric $C Y P 11 B 2 / C Y P 11 B 1$ gene with a novel breakpoint (g.9559-9742) on one allele, which differs from previous reports. Interestingly, the other allele contained a new disease-causing mutation, p.L340P, which altered the free energy and stability of $11 \beta$-hydroxylase. The chimeric mutation leads to deletion of CYP11B1 exons 1 to 6 on one allele, and unfortunately, a pathogenic missense mutation appeared on exon 6 of CYP11B1 in the other allele, which resulted in a seemingly homozygous missense mutation that was predicted by bioinformatics and molecular dynamics simulation to be a diseasecausing mutation. At present, only one case of a pathogenic p.G314R mutation combined with a nonfunctional chimeric CYP11B2/CYP11B1 has been reported [6]. Our pedigree analysis revealed that the proband carried both the chimera and p.L340P, and their coexistence increased the morbidity of $11 \mathrm{OHD}$ in this family. Our results suggest that chimera screening and CYP11B1 mutation screening should be simultaneously conducted and that pedigree study is necessary.

\section{Additional files}

Additional file 1: Table S1. The laboratory and endocrinological evaluation of the proband pre- and post-treatment. (PDF $200 \mathrm{~kb}$ )

Additional file 2: Table S2. Bioinformatics analysis of the free energy alteration in CYP11B1mut (PDF $82 \mathrm{~kb})$

Additional file 3: Figure S1. Graphs of sequence copy number around the mutation and multiple genes resulting in $\mathrm{CAH}$ of the proband and his mother by QPCR and targeted next-generation sequencing. (TIF $11910 \mathrm{~kb}$ )

Additional file 4: Figure S2. The sequencing chromatogram near the mutation in the proband's relatives. The box indicates the mutation location. (TIF $28380 \mathrm{~kb}$ )
Additional file 5: Figure S3. Radiation imaging of adrenal gland after treatment. A and B present abdominal $C T$ images after treatment for 6 months showing that the sizes of the left and right adrenal gland are $75.2 \mathrm{~mm} \times 22.1 \mathrm{~mm}$ and $67.3 \mathrm{~mm} \times 38.7 \mathrm{~mm}$, respectively. Similarly, C and $D$ present abdominal $C T$ images after treatment for 9 months showing that the sizes of the left and right adrenal gland are $65.2 \mathrm{~mm} \times$ $24.4 \mathrm{~mm}$ and $63.7 \mathrm{~mm} \times 35.7 \mathrm{~mm}$, respectively. The arrow shows the bilateral adrenal lesions. (TIF $63145 \mathrm{~kb}$ )

Additional file 6: Main methods used in the study. (DOC $112 \mathrm{~kb}$ ) Additional file 7: Consent form from the mother of the proband. (PDF $95.7 \mathrm{~kb})$

\section{Abbreviations}

11OHD: 11ß-hydroxylase deficiency; 17 KS: 17-ketosteroid; 17OHP: 17hydroxyprogesterone; ACTH: Adrenocorticotrophic hormone; ALD: Serum aldosterone; CAH: Congenital adrenal hyperplasia; CYP11B1mut: Mutation p.L340P; DOC: 11-deoxycorticosterne; SNPs: Single nucleotide polymorphisms

\section{Acknowledgments}

The authors would like to thank all the participants in this study, particularly Hongxia Wang, Xu Zhang and Honghe Fang for sequencing and bioinformatics assistance.

\section{Funding}

The collection of clinical data, data analysis and interpretation, and writing of the manuscript were supported by grants from the National Key R\&D Program of China (No. 2016YFC1101100), National Natural Science Foundation of China (NO. 81471039, No. 81270893, No. 81228023, No. 81401601, and No.81402202), Natural Science Foundation Project of Chongqing (CSTC2014jcyjjq10006, CSTC2012jjB10023, and

CSTC2016jcyjA0518), and Clinical Research Project of Xinqiao Hospital of Third Military Medical University (Yclkt-201421).

\section{Availability of data and materials}

All data generated or analyzed during this study are included in the article.

\section{Authors' contributions}

L.D. and RF.S.: data acquisition, analysis and interpretation; LY.S.: PCR assays; HT.Z. and Y.L.: study concept and design, analysis and interpretation of data, drafting of the manuscript, critical revision of the manuscript for important intellectual content, study funding, and study supervision. All authors have read and approved the manuscript for publication.

Ethics approval and consent to participate Not Applicable.

\section{Consent for publication}

Written informed consent was obtained from the patient's mother for publication of this case report and any accompanying images.

\section{Competing interests}

The authors declare that they have no competing interests. 


\section{Publisher's Note}

Springer Nature remains neutral with regard to jurisdictional claims in published maps and institutional affiliations.

\section{Author details}

'Department of Endocrinology, Xinqiao Hospital, Third Military Medical University, Chongqing 400037, China. ${ }^{2}$ Department of Endocrinology, Armed Police Hospital of Chongqing, Chongqing 400061, China.

Received: 14 December 2017 Accepted: 12 April 2018

Published online: 27 April 2018

\section{References}

1. Khattab A, Haider S, Kumar A, Dhawan S, Alam D, Romero R, et al. Clinical, genetic, and structural basis of congenital adrenal hyperplasia due to 11 betahydroxylase deficiency. Proc Natl Acad Sci U S A. 2017;114(10):E1933-40.

2. Soldin SJ, Soldin OP. Steroid hormone analysis by tandem mass spectrometry. Clin Chem. 2009;55(6):1061-6.

3. Auchus RJ, Arlt W. Approach to the patient: the adult with congenital adrenal hyperplasia. J Clin Endocrinol Metab. 2013;98(7):2645-55.

4. Hampf M, Dao NT, Hoan NT, Bernhardt R. Unequal crossing-over between aldosterone synthase and 11 beta-hydroxylase genes causes congenital adrenal hyperplasia. J Clin Endocrinol Metab. 2001;86(9):4445-52.

5. Portrat S, Mulatero P, Curnow KM, Chaussain JL, Morel Y, Pascoe L. Deletion hybrid genes, due to unequal crossing over between CYP11B1 (11 betahydroxylase) and CYP11B2(aldosterone synthase) cause steroid 11 betahydroxylase deficiency and congenital adrenal hyperplasia. J Clin Endocrinol Metab. 2001:86(7):3197-201.

6. Kuribayashi I, Nomoto S, Massa G, Oostdijk W, Wit JM, Wolffenbuttel BH, et al. Steroid 11-beta-hydroxylase deficiency caused by compound heterozygosity for a novel mutation, p.G314R, in one CYP11B1 allele, and a chimeric CYP11B2/CYP11B1 in the other allele. Horm Res. 2005;63(6):284-93.

7. Menabo S, Boccassini S, Gambineri A, Balsamo A, Pasquali R, Prontera O, et al. Improving the diagnosis of 11 beta-hydroxylase deficiency using homemade MLPA probes: identification of a novel chimeric CYP11B2/CYP11B1 gene in a Sicilian patient. J Endocrinol Investig. 2016;39(3):291-5.

8. Xu L, Xia W, Wu X, Wang X, Zhao L, Nie M. Chimeric CYP11B2/CYP11B1 causing 11 beta-hydroxylase deficiency in Chinese patients with congenital adrenal hyperplasia. Steroids. 2015;101:51-5.

9. Gu C, Tan H, Yang J, Lu Y, Ma Y. Congenital adrenal hyperplasia due to 11hydroxylase deficiency-compound heterozygous mutations of a prevalent and two novel CYP11B1 mutations. Gene. 2017;626:89-94.

10. El-Maouche D, Arlt W, Merke DP. Congenital adrenal hyperplasia. Lancet. 2017:390(10108):2194-210

11. Long $Y$, Han S, Zhang $X$, Zhang $X$, Chen $T$, Gao Y, et al. The combination of a novel 2 bp deletion mutation and p.D63H in CYP11B1 cause congenital adrenal hyperplasia due to steroid 11 beta-hydroxylase deficiency. Endocr J. 2016;63(3):301-10

12. Bulsari K, Falhammar H. Clinical perspectives in congenital adrenal hyperplasia due to 11 beta-hydroxylase deficiency. Endocrine. 2017;55(1):19-36.

\section{Ready to submit your research? Choose BMC and benefit from}

- fast, convenient online submission

- thorough peer review by experienced researchers in your field

- rapid publication on acceptance

- support for research data, including large and complex data types

- gold Open Access which fosters wider collaboration and increased citations - maximum visibility for your research: over $100 \mathrm{M}$ website views per year 